\title{
The Global Image of Shenzhen: A Corpus-Based Critical Discourse Analysis of Media Discourses
}

\author{
Zhide $\mathrm{Hou}^{1}$ \\ ${ }^{1}$ Shenzhen Tourism College of Jinan University, Shenzhen, China \\ Correspondence: Zhide Hou, Shenzhen Tourism College of Jinan University, Shenzhen, Guangdong 518053, \\ China. E-mail: zidhou@jnu.edu.cn
}

Received: November 2, 2018

Accepted: November 30, 2018 Online Published: December 29, 2018

doi:10.5539/ijel.v9n1p169

URL: https://doi.org/10.5539/ijel.v9n1p169

\begin{abstract}
This article studies the media representations of Shenzhen's global image by adopting the corpus-based critical discourse analysis. Previous studies mainly analyze the shaping, publicity and construction of Shenzhen's image from the perspective of urban culture and development and news dissemination. However, media construction on the global image of Shenzhen is empirical and noticeable. The findings demonstrate favorable representations of Shenzhen's image associated with technology powerhouse, manufacturing heartland, industrial boomtown, jewelry fair advantages and giant headquarters economy in general. Negative representations associated with Shenzhen Stock trading drop, landslide accident and Apple's Foxconn problems are amplified in media discourses.
\end{abstract}

Keywords: Shenzhen, global image, Corpus-based critical discourse analysis, media discourses, NOW corpus

\section{Introduction}

Shenzhen, designated as China's first Special Economic Zone in 1980, has been developed into a highlight of first-tier cities in terms of comprehensive competitiveness in China. Previously a small fishing village neighboring the Pearl River Delta and Hong Kong, Shenzhen has becoming one of the fastest-growing cities in the world for its vibrant economy since the institution of the stable policy of "Reform and Opening-up to the Outside World" in 1978. With its leading enterprises including Huawei, the internet giant Tencent and alongside emerging innovative small and medium sized enterprises, Shenzhen displayed a global image of an international innovative hub. One of the City slogans saying "Shenzhen is home to all who resides" added to its popularity as a melting pot of China. It was due to its inclusive culture, Shenzhen has shaped a national image of home to young migrants with more job opportunities.

To date, studies on Shenzhen's global image are limited among Chinese academics in four aspects. First, the positive culture and communication effects are articulated due to the $26^{\text {th }}$ Summer Universiade held in Shenzhen (Chen, 2011; Wu, 2012; Pan, 2013). Second, Li \& Liu note the construction, development and promotion of Shenzhen's global image as seashore tourism (Li, 2003; Liu, 2011). Third, Huang (2007) explains the literary construction of Shenzhen's multi-dimensional narrative integration. Last, Liu (2013) conducts a textual analysis to the ideological Shenzhen-related reporting and global image of the news reports from the New York Times. Previous studies mainly analyze the shaping, publicity and construction of Shenzhen's image from the perspective of urban culture and development and news dissemination. However, empirical study of discursive construction on the global image of Shenzhen associated with media discourses is not systematic and comprehensive enough. It is thus valuable to investigate media representations of Shenzhen's global image so as to enhance city image and cultural soft power. To fill the gap, the present study adopts a corpus-based critical discourse analysis (hereafter CDA) (Baker et al., 2008; Brindle, 2016; Cheng \& Lam, 2013; Kim, 2014) of media discourses, in order to answer the following research questions:

- In what ways are "Shenzhen" linguistically defined and constructed?

- What are the frequent topics discussed in news reports relating to "Shenzhen"?

- What attitudes towards "Shenzhen" emerge from the corpus of media discourses?

To achieve the research objectives, section 2 is devoted to literature review of utilizing analytical framework of corpus-based CDA. Section 3 gives a description of the data and methodology. Section 4 reports findings of five 
semantic sets generated from the data. Section 5 summarizes the major findings of the study and underscores the contribution of the useful methodological synergy by combing corpus linguistics (hereafter CL) and CDA.

\section{Corpus-Based Critical Discourse Analysis}

The most basic aspect of corpus-based approach is informed by the frequency of occurrence of words in a corpus. Keyness and collocations are the two main contributions which determine words that are significantly more frequent and meaningful and examine "a semantic analysis of a word" (Sinclair 1991, pp. 115-116). For keyword keyness, it is defined as the statistically significant higher frequency of particular words or clusters in the study corpus in comparison with reference corpus. The identification of keywords can indicate what a corpus is about, the "aboutness" of a corpus. Keywords can also be used to refer to lexical items that are closely related to the representation of ideology (Adolphs, 2006). For collocational analysis, it is statistical calculation of collocation based on three measures: the frequency of the node, the frequency of the collocates, and the frequency of the collocation (Baker et al., 2008, p.278).

The paradigm of CDA was developed by van Dijk (1993), Fairclough (1995) and Wodak et al. (1999) and sees language as social practice and considers the context of language use to be crucial. The relationship between discursive acts and the contextual and social structures in which they are embedded is dialectical. This means discourse constitutes social practice and is at the same time constituted by it. Blommaert \& Bulcean (2000, p. 451) notes that "CDA conceives discourse as a social phenomenon and seeks to improve the social-theoretical foundations for practicing discourse analysis as well as for situating discourse in society". The aim of CDA is to intervene discursively in given social practice, making certain social situations more transparent through linguistic evidence.

Corpus approach of CDA holds a hegemonic position to and has produced the majority of research in media discourse studies. It has arguably become the standard framework for studying media discourses. The "CDA journal" (Garrett \& Bell, 1998, p. 6) Discourse and Society deals with voluminous media discourses by adopting corpus approach of CDA as an analytical framework. The methodological synergy between CL and CDA serves as a powerful tool to deconstruct and analyze media discourses.

Johnson et al. (2003) explore discourses of "political correctness" in a corpus of articles gathered from three broadsheet newspapers in UK between 1994 and 1999. Focusing on the keywords of the discourse in which "PC"-related terms appear, they examine the five expressions involving political correctness, political incorrectness, politically correct, politically incorrect and "PC", followed by compilation of the keywords which occurred most frequently within the corpus in relation to the term "political correctness". Findings show the shifts in which discourses of "political correctness" have been drawn upon as a means of framing debates over the British Labour Party. Page (2003) examines the complex and contradictory patterns of representation of Cherie Booth/Blair, wife of former British Prime Minister Tony Blair, from a corpus of 130 press reports from 1997 to 2002. Focusing on naming practices and transitivity choices and a frequency-based analysis of collocational patterns related to the phrase "working mother", the author discussed the textual heterogeneity as symptomatic of social change. Findings show the reinforcement of the gendered inequality presupposed by the ideological model of separate spheres.

Moreover, Baker et al. (2008) adopt a corpus-based approach to examine a 140-million-word corpus of British news articles about refugees, asylum seekers, immigrants, and migrants from 1996 to 2005 . They analyze their lexical patterns by focusing on keyness, collocation and concordances. Their project supports substantially that the CL analysis can overlap with that of CDA. Hakam $(2009$, p. 33) explores a corpus of 422 "hard" news texts from the websites of 19 English-language Arab newspapers that "address the events and issues known as the Prophet Muhammad cartoons controversy". By adopting a corpus-based approach to the analysis of collocational profile of "cartoons", Hakam (2009) concludes that discourse generated by the "cartoons controversy" proved to be a locus of ideological struggle rooted in socio-cultural conflicts. Caldas-Coulthard and Moon (2010) adopt a corpus-based approach to explore the adjective lexicon of the tabloid press in comparison with quality newspapers in order to investigate how social actors are classified in the public discourse of the media. Findings show that the discursive representation of "curvy, hunky, kinky are not just salacious labels of the tabloid press, nor are old, tall, strong, pretty, wild simple descriptors of the objects of newspaper reporting (p. 125)", indicating that the media categorizes people through very specific points of view and values not always apparent to a non-critical reader. Koteyko (2010) adopts the corpus-based approach to explore a corpus of 82,049 RSS (Really Simple Syndication) feeds, the web-based summaries from blogs, news sites and other sources concerning the online dimension of the interpretative struggle around the issue of climate mitigation. Focusing on the concordances analyses of "carbon keywords" of finance terms (e.g. carbon trading), popular terms (e.g. carbon 
footprint), and dramatic terms (e.g. carbon guilt), the author reveals "semantic associations surrounding the compounds and highlights connotational differences that signal both support and criticism of the climate mitigation initiatives proposed by policy makers and environmentalists" (p. 655). Prentice (2010) examines a 3,351,883-word corpus built from an internet discussion forum on Scottish pro-independence. The author compares the corpus under investigation with a larger reference corpus LexisNexis database, British newspaper articles on Scottish independence. Findings show that "Scottish independence will strengthen, consolidate or transform Scottish identity in a positive way and a distinct lack of strategies that seek to dismantle British identity or refer to historical disputes" (p. 405).

Furthermore, Jaworska and Krishnamurthy (2012) conduct a corpus-based discourse analysis of the search term feminism in the British and German contexts in order to avoid the marginalized feminism in the media arena. The authors examine the representations of feminism in the reference corpora-Bank of English, a corpus created by COBUILD, and Institut fur Deutsche Sprache (IDS), the largest collection of texts in German. Findings show feminism in both reference corpora is framed predominantly as a political movement closely associated with radicalism, militancy, and leftist ideology, which in turn have distinctively negative connotations. Then they examine the representations of feminism in the study corpora-British and German press corpora, revealing that there is a strong tendency to portray the movement in negative terms and their association with countries of the western world, but not implying radicalism, demonism or charged circumstances which are evident in the analysis of the two reference corpora. Fusari (2013) examines the crisis and privatization of the Italian airline, Alitalia in a set of newspaper corpora published by Italian, British and American broadsheet press. By focusing on the interlinguistic and intralinguistic comparison of the language of the broadsheet press among the three press corpora, the author finds the British corpus has most aggressive forms of verbal irony against the violations of market principles, the Italian corpus "resorts to irony in its criticism of governmental measures...not the violation of free market rules" (p. 330), while the American corpus has less verbal irony given other discourse strategies are used.

Additionally, more recent research can be found in Kim (2014) who examines the US mainstream media discourses on North Korea through the corpus-based CDA approach to find that the US media divide the world according to the countries' political position towards the USA. Camiciottoli (2016) identifies the compounds of self-expression in the blog engaging in conversations about fashion through a corpus approach, to find how bloggers use creative language to construct their identities as members of a distinctive and cohesive social community. Brindle (2016) critically compares the discursive constructions of the Sunflower Student Movement in Taiwan through the corpora of two major English-language newspapers. Focusing on the analysis of the frequency lists and keywords, the author provides evidence of ideological differences in patterns of language use produced by the two newspapers.

The above literature reviewed provides strong evidence of the methodological synergy with CL and CDA combined to explore effectively the discursive events of politics, government, religion, refugees, identity, conflicts and feminism. The advantage of integrating CL and CDA has mostly been used for the analysis of media and political discourse. With a reasonably high degree of objectivity, CL bolstering CDA can provide reliable generalizations about language use. Analysts can go beyond single text and conveniently explore quantitative patterns in a large number of texts before carrying out qualitative analysis. Given the methodological power of marrying CL and CDA, the present study adopts the corpus-based quantitative analyses of frequency data in combination with qualitative critical discourse analysis, in order to uncover the media discursive construction of Shenzhen.

\section{Methodology}

The data of this study adopts the NOW corpus (News on the Web) as the study corpus to investigating the global image of Shenzhen. Containing 6.64 billion words of data from web-based newspapers and magazines from 2010 to the present time November 2018, the NOW corpus is a user-friendly resource developed by professor Mark Davies from Brigham Young University (https://corpus.byu.edu/now/). The NOW corpus is valuable for the purpose of the present study because it consists of texts featured in most common sources such as Huffington Post, NPR, CNN, The Atlantic, TIME, Los Angeles times, Wall Street Journal, Slate Magazine, USA Today, ABC News from 20 English speaking countries or regions. It is also valuable to provide a more comprehensive analysis of the global image of the Shenzhen given the NOW corpus covers the time span of almost nine years (January 2010-November 2018) which is good for media representations of Shenzhen since its establishment as China's first special economic zone for thirty years (1980-2010). In particular, this period also covers the $26^{\text {th }}$ summer Universiade held in Shenzhen in 2011 which might be the hot spot of international media coverage. 
Accompanied the BYU interface, the NOW corpus provide a search box on the left-hand side of the interface with the main functions of displaying list, chart and collocates of the very word users typed in. For the first step, the search word "Shenzhen" was typed in by generating a frequency of 20,071 times in the NOW corpus, which is an indication of desirable corpus for the present study. For the second step, after choosing the collocates function and typing in the search word "Shenzhen" and asterisk * as collocates, I retrieved a list of words collocating with "Shenzhen" in order of frequency. This is normally done by the default setting of four words on both sides of the node for generating the collocates. As Sinclair et al. (2004) notes that "the patterning is a little stronger on the left than on the right", I set a span of five words on the left and four words on the right of the node "Shenzhen". The collocates of "Shenzhen" provides an important part of the patterning of meanings associated with the discursive construction of Shenzhen. The last step involves manual picking the top items from the collocate lists for concordance analysis in five semantic sets. At this point, Sinclair's (2004) model of lexical description comprising mainly semantic preference and semantic prosody are utilized for concordance analysis of the collocates of "Shenzhen". Semantic preference is "the restriction of regular co-occurrence of items which share a semantic feature" (Sinclair 2004: 142) and semantic prosody is "the determiner of the meaning of the whole lexical item" (Sinclair 2004: 141).

Clearly, CDA plays an important role which goes insight into the ideologies of discourse processing at the corpus-based approach of discursive constructing the image of Shenzhen.

\section{Discussion of Findings}

Among the top 100 items, the list of collocates associated with the search word "Shenzhen" appeared to be categorized into five main semantic sets including "name of cities", "technology", "trading and manufacturing", "headquarters" and "name of companies", as Table 1 shows.

Table 1. The collocates of "Shenzhen" in the top 100 collocate list categorized in five semantic sets.

\begin{tabular}{|c|c|c|c|c|}
\hline Rank order & Collocates & Frequency & $\%$ & Semantic sets \\
\hline 2 & Shanghai & 2036 & 1.92 & \multirow{20}{*}{ Name of cities } \\
\hline 5 & Hong & 1062 & 0.27 & \\
\hline 6 & Kong & 1010 & 0.27 & \\
\hline 8 & Guangzhou & 896 & 5.22 & \\
\hline 10 & Beijing & 737 & 0.29 & \\
\hline 28 & Hangzhou & 174 & 2.05 & \\
\hline 35 & Chengdu & 138 & 1.77 & \\
\hline 37 & Tianjin & 136 & 1.32 & \\
\hline 48 & Wuhan & 98 & 1.67 & \\
\hline 49 & Zhuhai & 97 & 4.25 & \\
\hline 50 & Macau & 96 & 0.24 & \\
\hline 53 & Dongguan & 89 & 6.57 & \\
\hline 59 & Xiamen & 70 & 1.59 & \\
\hline 60 & Nanjing & 70 & 1.21 & \\
\hline 70 & Chongqing & 58 & 0.76 & \\
\hline 72 & Qingdao & 57 & 1.15 & \\
\hline 76 & Foshan & 55 & 6.67 & \\
\hline 92 & Suzhou & 45 & 1.63 & \\
\hline 97 & Tokyo & 43 & 0.03 & \\
\hline 98 & Huizhou & 42 & 10.63 & \\
\hline 18 & Technology & 392 & 0.02 & \multirow{2}{*}{ Technology } \\
\hline 54 & Tech & 87 & 0.02 & \\
\hline 27 & Trading & 176 & 0.03 & \multirow{7}{*}{ Trading and Manufacturing } \\
\hline 29 & Factory & 165 & 0.08 & \\
\hline 30 & Manufacturing & 162 & 0.04 & \\
\hline 33 & Industrial & 160 & 0.04 & \\
\hline 83 & Factories & 51 & 0.07 & \\
\hline 86 & Jewelry & 50 & 0.06 & \\
\hline 100 & Manufacturer & 42 & 0.04 & \\
\hline 32 & Headquarters & 160 & 0.06 & \multirow{2}{*}{ Headquarters } \\
\hline 55 & Headquartered & 81 & 0.21 & \\
\hline 36 & Foxconn & 137 & 1.50 & \multirow{3}{*}{ Name of companies } \\
\hline 45 & Huawei & 126 & 0.24 & \\
\hline 68 & Tencent & 59 & 0.29 & \\
\hline
\end{tabular}




\subsection{The City of Shenzhen}

For name of cities, Table 1 lists a collection of cities that appear in the top 100 collocate list, ordered according to frequency. It displays a number of Chinese cities except Tokyo, the Japanese capital city. Shanghai, China's largest city that co-occurs with Shenzhen in media discourses, has shown three recurrent patterns: "Shanghai Stock Exchange and Shenzhen Stock Exchange", "Shanghai Composite index and Shenzhen Composite index" and "first/second-tier cities in China including Shanghai, Shenzhen and others". Thus, Shenzhen seems to have the same financial position as Shanghai does given one of the stock exchanges located in Shenzhen. China's big cities such as Shanghai and Shenzhen always go hand in hand in news reports for their economic and trading status.

The collocation "Hong" and "Kong" should be combined as "Hong Kong" due to the limitation of searching one word for collocate. The concordance lines of this collocate shows a high percentage of three collocational patterns such as "Hong Kong, Guangzhou and Shenzhen", "Hong Kong, Shanghai and Shenzhen" and "Hong Kong, Macau and Shenzhen", indicating a relationship of geographical connection with Guangzhou and Macau and economic vibrance with Shanghai.

Other mainland cities collocate with Shenzhen are found to be in association with areas such as Artificial Intelligence development, highest level of intensification, car park payment system, innovative tech, production centers, big spenders, economically developed, super-rich (for three cities Shanghai, Hangzhou and Shenzhen), manufacturing hub, leading technology, government incentive programmes, potential investors, industry center, and etc., all of which provide a positive semantic prosody of representing Shenzhen.

Among the collocate list of cities, the most striking pattern revealed is that "Shenzhen" collocates with "Tokyo". The concordance analysis shows that Shenzhen has been reported as one of the international cities in the recurrent patterns with Tokyo, and many other international cities such as Hong Kong, New York, Paris, Bangkok, New Delhi, Singapore, Miami, Istanbul, Sydney and Seoul associated in the field of bourse, clinical laboratories, headquarter offices, eco-system, industrial hub, investment and Asian tiger centers, as the following sample concordance lines shows (figure 1):

\footnotetext{
, FDA-registered, clinical laboratory in New Jersey and licensed laboratories in Hong Kong, Shenzhen, China and Tokyo, Japan, operating on our proprietary platform, dedicated to CITIC Capital is headquartered in Hong Kong, with offices in Shanghai, Beijing, Shenzhen, Tokyo, New York, with a team of 280 staff. \# CITIC

have to share the spotlight with the emerging eco-systems of Berlin, Los Angeles, Shenzhen, Paris and Tokyo; traditional tour operators will start innovating in interesting ways; the ATP Finals title in London last week, he also claimed back-to-back titles at Shenzhen and Tokyo. \# He was able to carry his great form to Lille, Grigor Dimitrov for the ATP Finals title in London, Goffin claimed back-to-back titles at Shenzhen and Tokyo. Next could be a Davis Cup triumph in the heated atmosphere of tournament as the seventh seed with two titles to his name - at Tokyo and Shenzhen - in a year which has seen him break into the top 10 for the

enjoyed the best season of his career, including back-to-back Tour titles in October in Shenzhen and Tokyo. \# Jack Sock \# Age: 25 \# World ranking: 9

the majority of them in Asian coastal megacities and industrial hubs such as Shanghai, Shenzhen, Bangkok and Tokyo. \# Japan's second biggest city, Osaka, is tops this sustainable index, followed by London in second and Tokyo in third. Shenzhen is at 35 th place by this measure, while Hong Kong is at 13 th place 8/6). \# David Goffin's winning run came to an end, the Shenzhen and Tokyo champion beaten 7-6 (7/4) 6-3 by Gilles Simon, while Kevin Toronto, Seattle, Shanghai, Beijing, Taiwan, Tokyo, Hong Kong, Shenzhen, Chengdu and Singapore, and over 25 billion dollars of projects complated or under
}

Figure 1. Sample concordance lines for Shenzhen/Tokyo

To conclude, Shenzhen is positively represented as one the national economic center and regional vibrant city. The collocation with a number of other mainland cities and the international city Tokyo is evident of Shenzhen's potential advantage and huge capacity both at home and abroad.

\subsection{The Technology of Shenzhen}

Of the 479 instances of the collocates with both "technology" and "tech", 328 concordance lines $(68 \%)$ show a strong recurrent pattern of "Shenzhen...Technology/Tech Co Ltd.", which provides a discursive construction of Shenzhen's high-tech empire in China. Other frequent recurrent patterns "technology/tech...of/in Shenzhen" are found to be the most significant, accounting for 70 concordance lines. Shenzhen's technological position and function are described as hub, powerhouse, center, headquarter, manufacturer and etc., as the following extract indicates: 
(Yahoo News, 14 January 2018)

Similarly, Chinese money is helping to build the Sihanoukville Special Economic Zone, which has its sights set on becoming the Cambodian equivalent of southern China's flourishing tech hub of Shenzhen, and has already attracted about 100 Chinese firms.

It should be noted that Shenzhen's successful development in technology is catching the world attention and cementing its place on the global stage as one of the major players in high-tech.

\subsection{The Trading and Manufacturing in Shenzhen}

A reading of the collocational pattern of "Shenzhen/trading" has shown the semantic preference of a strong linkage with Shanghai and Hong Kong to which the stock exchanges connect. This is an indication of a unique collaboration between Hong Kong, Shanghai and Shenzhen Stock Exchanges. Regarding the semantic prosody, $17 \%$ (30 out of 176) of the instances carry the negative meaning of "falling" of Shenzhen Stock Exchange. This is because this pattern is co-selected with words such as decline, slid, dropped, fell, halt, tumbled, and so on.

The collocational pattern of "Shenzhen/manufacturing" shows the semantic preference of Shenzhen's position as manufacturing center. This is because the 74 concordance lines (46\%) out of the 162 instances are found in the recurrent pattern of "manufacturing * of/in Shenzhen". The asterisk is used to refer to such attributive nouns including hub, bases, plant, facilities, factory, partner, heartland, mecca, boomtown, center, campus, metropolis, backbone, epicenter and power and so on. Syntagmatically, the consistent pattern of co-selections, such as good synergy, strong, advanced, excellence, fast-growing, grown significantly, suitable city, well-established, boomtown, fast-growing, fast-paced and development in 9\% (15 out of 162) of the instances, serves to create the positive semantic prosody of favorable manufacturing environment in Shenzhen, as the following extract shows:

(The Asian Age, 21 May 2016)

Innovative new companies are drawn by Shenzhen's well-established manufacturing supply chains and transport links, proximity to Hong Kong's banking and financial expertise, and better traffic, milder weather and less air pollution than Beijing and Shanghai.

The collocational pattern of "Shenzhen/industrial" is found to be in frequent association with "park" $(22 \%, 35$ out of 160). An examination of the concordance lines identifies the co-selection with "landslide" that hits an industrial park in Shenzhen. It is a tragedy caused by the collapse of a huge pile of construction waste, rather than any natural geological phenomenon. Shenzhen's landslide accident seems to be amplified in media discursive construction given its general image is described as a model, modern city for its industrial advantages associated with words such as boomtown, ecosystem, revolution, hub, and heartland, as the extract shows:

(The Atlantic, 5 June 2016)

But it was mainly the speed of the industrial ecosystem in places like Shenzhen that was so amazing. And the density of all elements of the supply chain - if you needed a certain kind of keyboard or connector, there were five suppliers to choose from within an hour's drive.

Lastly, the collocate with "jewelry" is found to be in the strong cluster of "Shenzhen Jewelry Fair" in 66\% (33 out of 50) of the instances. Started from 1980s, Shenzhen jewelry industry leads in mainland China and has become the biggest gold jewelry manufacturing base and trade distribution center since 1990s. The concordance analysis shows Shenzhen jewelry fair is favorably represented for its advantages in aesthetics, organizer, fashion elements, networking opportunities, efficient platform, sustainable development, number of exhibitors, new features, and manufacturing excellence.

\subsection{Headquarters in Shenzhen}

The collocational pattern with both "headquarters" and "headquartered" provide evidence that Shenzhen's most important economic sector lies in its role as headquarters base of many China's high-tech companies. As home to the headquarters of numerous multinational companies, Shenzhen exhibits a vibrant and expanded regional advantage in the Pearl River Delta area, as the following extract indicates:

(Travel Daily News, 25 October 2017)

According to the Los Angeles County Economic Development Corporation (LAEDC), majority of Chinese investment into the United States originates from Guangdong Province which covers Shenzhen and Guangzhou. Dr. Chi added, "At no time is the connectivity between these two cities more needed than it is now when China's homegrown tech companies with headquarters in Shenzhen are expanding their presence and direct investments in the United States". 


\subsection{Shenzhen Companies in the World}

The most frequent collocates with "Foxconn" in 137 instances is identified in the recurrent pattern to be associated with "iPhone" or Apple-affiliated businesses in 16\% (22 out of 137) of the instances. An examination of the other concordance lines shows the Apple's Foxconn problems which is evident from the co-selected words or phrases of this collocational pattern such as heavy nose bleeds, suicides, work overtime, complaints, ultra-low wages, repetitive, exhausting and alienating, killed, died, depressing and regime, as the following extract indicates:

(BBC News, 18 May 2010)

Foxconn, the Taiwanese maker of the Apple iPhone, has hired counsellors and brought in Buddhist monks for its workers after a spate of suicides. This year, seven employees have killed themselves and two have tried. The deaths, at Foxconn's manufacturing campus in Shenzhen, southern China, have raised concerns about worker treatment. Foxconn is part of Hon Hai Precision, the world's largest maker of consumer electronics. It is also one of the biggest overseas employers in China.

Known for assembling famous electronic goods like Apple's iPhone, Foxconn in media reports gained a dark image as a place where distraught workers regularly throw themselves to their deaths.

Next, the frequent collocational pattern "Shenzhen/Huawei" is identified to be frequent in association with "headquarter" ( $42 \%, 53$ out of 126) and "technology" ( $8 \%, 10$ out of 126). Huawei, a leading global provider of information and communications technology, headquartered in Shenzhen with a strong recurrent pattern represents a deserved value for Shenzhen's image as an emerging tech city. Huawei technology is gaining momentum in IT development in the world, with its smartphone technology has unseated Apple to become the world's second-largest smartphone maker. The following extract indicates Huawei's cutting-edge innovative technology:

\section{(Irish Independent, 18 August 2018)}

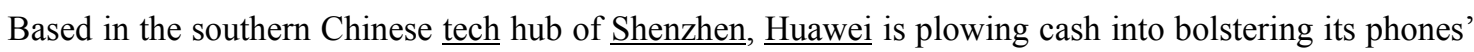
camera capabilities in a quest to dominate. Its flagship P20 Pro boasts a three-lens camera that was co-engineered with the 104-year-old German camera maker Leica. Huawei also offers a shiny, rainbow-effect handset finish known as Twilight, differentiating their product from the many monotone smartphones on the market.

Tencent, one of the world's largest internet companies, co-occurs with "Shenzhen" in 59 instances and shows three most frequent recurrent patterns, "Tencent founded/based/headquartered in Shenzhen". Like Huawei, Tencent is also headquartered in Shenzhen and is becoming one of the world's most valuable technology companies. Together with Huawei, Tencent is forging China's equivalent of Silicon Valley in Shenzhen. As home to the two tech giants, Shenzhen symbolizes the window for advances in technology.

\section{Conclusion}

By conducting a corpus-based CDA that integrates CL and CDA, this study has demonstrated how the two can be effectively combined and the great potential of using NOW corpus. Through generating the collocate list of "Shenzhen" and the classification into five semantic sets, this study has shown Shenzhen's city image of regional and national economic power and international developmental capacity. The global image of Shenzhen is favorably represented for its technology powerhouse, manufacturing heartland, industrial boomtown, jewelry fair advantages and giant headquarters economy in general. However, negative representations associated with Shenzhen Stock trading drop, landslide accident and Apple's Foxconn problems are amplified in media construction. These findings not only give insights into the global image of Shenzhen, but also provide linguistic evidence to the perception of Shenzhen identity.

\section{Acknowledgements}

The work described in this paper was substantially supported by the Project of Philosophy and Social Sciences Sponsored by Shenzhen Association of Social Sciences (Project No.: SZ2018B025).

\section{References}

Adolphs, S. (2006). Introducing electronic text analysis: A practical guide for language and literary studies. New York: Routledge.

Baker, P., Gabrielatos, C., Khosravinik, M., Krzyzanowski, M., McEnery, T., \& Wodak, R. (2008). A useful methodological synergy? Combining critical discourse analysis and corpus linguistics to examine 
discourses of refugees and asylum seekers in the UK press. Discourse \& Society, 19(3), 273-306. https://doi.org/ 10.1177/0957926508088962

Blommaert, J., \& Bulcean, C. (2000). Critical discourse analysis. Annual Review of Anthropology, 29, 447-66. https://doi.org/10.1017/CBO9780511610295.003

Brindle, A. (2016). A corpus analysis of discursive constructions of the Sunflower Student Movement in the

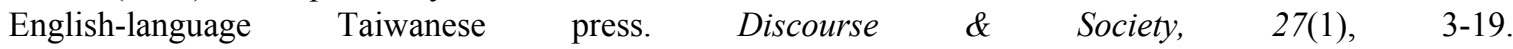
https://doi.org/10.1177/0957926515605957

Caldas-Coulthard, C. R., \& Moon, R. (2010). 'Curvy, hinky, kinky': Using corpora as tools for critical analysis. Discourse \& Society, 21(2), 99-133. https://doi.org/10.1177/0957926509353843

Camiciottoli, B. C. (2016) “All those Elvis-meets-golf-player looks": A corpus-assisted analysis of creative compounds in fashion blogging. Discourse, Context \& Media, 12, 77-86. https://doi.org/10.1016/j.dcm.2015.10.002

Cheng, W., \& Lam, P. W. Y. (2013). Western perceptions of Hong Kong ten years on: a corpus-driven critical discourse study. Applied Linguistics, 34(2), 173-90. https://doi.org/10.1093/applin/ams038

Cheng, Z. (2011). World University games and the Shenzhen cultural image. Journal of Guangzhou Sport University, 31(4).

Fairclough, N. (1995). Media Discourse. London: Arnold

Fusari, S. (2013). 'A miracle. Madonna!': Irony in the representation of Alitalia's privatization by the Italian, British and American press. Discourse and Society, 24(3), 315-333. https://doi.org/10.1177/0957926512471761

Garrett, P., \& Bell, A. (1998). Media and discourse: A critical overview. In A. Bell \& P. Garrett (Eds.), Approaches to media discourse. Malden: Blackwell.

Hakam, J. (2009). The 'cartoons controversy': A critical discourse analysis of English-language Arab newspaper discourse. Discourse and Society, 20(1), 33-57. https://doi.org/10.1177/0957926508097094

Huang, Y. (2007). Shenzhen's narration and the city's image. Journal of Shenzhen University (Humanities \& Social Sciences), 24(4).

Jaworska, S., \& Krishnamurthy, R. (2012). On the F word: A corpus-based analysis of the media representation of feminism in British and German press discourse, 1990-2009. Discourse \& Society, 23(4), 401-431. https://doi.org/10.1177/0957926512441113

Johnson, S., Jonathan, C., \& Stephanie, S. (2003). From "politically correct councilors" to "Blairite nonsense": Discourse of "political correctness" in three British newspapers. Discourse \& Society, 14(1), 29-47. https://doi.org/10.1177/0957926503014001928

Kim, K. H. (2014). Examining US news media discourses about North Korea: A corpus-based critical discourse analysis. Discourse \& Society, 25(2), 221-244. https://doi.org/10.1177/0957926513516043

Koteyko, N. (2010). Mining the internet for linguistic and social data: An analysis of 'carbon compounds' in web feeds. Discourse and Society, 21(6), 655-674. https://doi.org/10.1177/0957926510381220

Li, L. (2003). The development and image construction of coastal tourism in Shenzhen. Theory and Practice of the Special Zone, 5.

Liu, P. (2013). The ideology and construction of "Shenzhen image" in: an example of 30-year's (1980-2010) Shenzhen-related reports in of the New York Times. Media Culture, 1.

Liu, X. (2011). Study on the construction and management of images of international seaside tourism city. Economic and Social Development, 9(5).

Page, R. E. (2003). 'Cherie: lawyer, wife, mum': Contradictory patterns of representation in media reports of Cherie Booth/Blair. Discourse and Society, 14(5), 559-579. https://doi.org/10.1177/09579265030145002

Pan, X. (2013). The strategy of government press release and dissemination of city image: an example of 2011 Universiade in Shenzhen. Academic Debate, 5.

Prentice, S. (2010). Using automated semantic tagging in critical discourse analysis: a case study on Scottish independence from a Scottish nationalist perspective. Discourse and Society, 21(4), 405-437. https://doi.org/ 10.1177/0957926510366198 
Sinclair, J. McH. (1991). Corpus, concordance, collocation. Oxford: Oxford University Press.

Sinclair, J. McH. (2004). Trust the text. Routledge: London.

van Dijk, T. A. (1993). Principles of critical discourse analysis. Discourse \& Society, 4(2), 249-83. https://doi.org/ 10.1177/0957926593004002006

Wodak, R., De Cillia, R., Reisigl, M., \& Liebhart, K. (1999). The discursive construction of national identity. Edinburgh, Scotland: Edinburgh University Press.

$\mathrm{Wu}$, J. (2012). The cultural effects of the Universiade and Shenzhen's urban image. Journal of Shenzhen University (Humanities \& Social Sciences), 29(1).

\section{Copyrights}

Copyright for this article is retained by the author, with first publication rights granted to the journal.

This is an open-access article distributed under the terms and conditions of the Creative Commons Attribution license (http://creativecommons.org/licenses/by/4.0/). 